\title{
ED chief complaint categories for a medical student curriculum
}

\author{
Adam R. Kuykendal • Judith Tintinalli • Kevin Biese
}

Received: 11 January 2008 / Accepted: 31 March 2008/Published online: 5 June 2008

(C) Springer-Verlag London Ltd 2008

\begin{abstract}
Background It is important to stimulate the interest of all medical students in emergency medicine to further its growth nationally and internationally. Students focused on other specialties can benefit from exposure to the more common, less acute problems seen in the emergency department (ED).

Aims We developed a categorization system for chief complaints (CC) in an academic ED fast track (FT) area, so that a curriculum based upon actual $\mathrm{CC}$ and clinical experience could be designed for learners.

Methods Primary (first) FTCCs were obtained from the ED electronic record of an academic medical center from 1 to 10 July 2006 and 1 to 10 February 2007. Category definitions were developed, and CCs were collapsed into clinically coherent groups. Inter-rater reliability was
\end{abstract}

Research was done while Dr. Kuykendal was a senior medical student at the University of North Carolina

Work was presented in May 2007 at the SAEM Annual Meeting in Chicago, IL with corresponding abstract publication in the May supplement of AEM.

The views expressed in this paper are those of the author(s) and not those of the editors, editorial board or publisher.

J. Tintinalli $(\bowtie) \cdot K$. Biese

Department of Emergency Medicine,

University of North Carolina School of Medicine,

CB 7594, Chapel Hill, NC 27599-7594, USA

e-mail: jet@med.unc.edu

Present address:

A. R. Kuykendal

Department of Internal Medicine,

Northwestern McGaw Medical Center,

1410 N. Cleveland Ave., Unit 3N,

Chicago, IL 60610, USA

e-mail: a-Kuykendal@md.northwestern.edu assessed, and CC categories were compared for the two study periods. The study was exempted by the University Institutional Review Board.

Results In the July data set, 493 CCs were placed into 8 categories which captured $96.3 \%$ of CCs: pain $32.3 \%$, injury $26.6 \%$, infection $15 \%$, psychiatric $8.1 \%$, miscellaneous $6.1 \%$ (those with a frequency of $\leq 2 \%$ ), nurse only visit $5.5 \%$, eye $4.3 \%$ and rash $2.2 \%$. The weighted kappa for CC categorization between two observers was 0.8980 (95\% confidence interval: $0.8638-0.9322$ ). In the February data set, refinements with 454 CCs resulted in categorization of $96.9 \%$ of the CCs into the same 8 categories with 53 subcategories. The distribution of the major categories was similar in July and February $(p=0.13)$.

Conclusions The FTCC categorization was valid and reliable and can guide curriculum development for learners in an academic setting.

Keywords Education · Curriculum

\section{Introduction}

It is important to stimulate the interest of all medical students in emergency medicine to further its growth nationally and internationally. Students focused on other specialties can benefit from exposure to the more common, less acute problems seen in the emergency department (ED). In our medical school, the ED fast track (EDFT) is one option available to medical students to fulfil an ambulatory care curriculum requirement.

We set out to categorize the EDFT patient 'reasons for visit', documented as free-text chief complaints (CCs), into clinically useful groupings. Our objective was to better understand the types and volumes of CCs in the EDFT and to assess the educational experience of our students. We 
aimed to develop a scheme that would allow us to classify $\mathrm{CCs}$ based upon actual clinical experience. A curriculum could then be developed starting with the $\mathrm{CC}$, and then moving onto the process of evaluation and decision making based upon the CC.

\section{Methods}

\section{Study design}

In this descriptive study, free-text CCs were examined by the lead author, who devised a clinically relevant, hierarchical categorization scheme. The scheme was refined and inter-rater reliability was determined. These data were also compared with free-text $\mathrm{CCs}$ analysed during a different time period. This study was exempted by the Institutional Review Board.

Study setting and population

The study took place in an academic medical center's EDFT. The EDFT volume is about 15,000 patients per year, $\geq 16$ years of age. FT operates from 9 a.m. to 2 a.m. and all patients presenting during those hours with an Emergency Severity Index (ESI) [1] triage category 4 or 5 (occasionally 3 ) are examined there. The study analysed the CCs of all patients seen in FT from 1 to 10 July 2006. A second analysis was done from 1 to 10 February 2007.

\section{Study protocol}

The triage nurse entered free-text $\mathrm{CC}$ as his or her interpretation of the patient's reason for the visit. When $>$ $1 \mathrm{CC}$ was entered, only the first $\mathrm{CC}$ was analysed. CCs that were uninterpretable were excluded from analysis. Examples of uninterpretable CCs were the following terms: 'jail', 'bone', 'legs'. From this list of CCs, the lead author developed a hierarchical categorization scheme using face validity. Subcategories were created if there were $>2$ complaints in a major category. Each major category had to adequately encompass its subcategories and contain $>2 \%$ of all CCs. When a CC did not meet the above criteria, it was classified as 'miscellaneous'. Face validity [2] was assessed and the scheme revised by the co-authors, two EM faculty members who are experts in emergency medicine medical education. Validity was assessed when CCs were categorized by two different individuals who were medical students on the ED rotation (see "Acknowledgements"), one using CCs from July 2006, and the other using CCs from February 2007. Inter-rater reliability was assessed by one medical student who was blinded to the categorizations, for data from July 2006.
Measurements and data analysis

Simple percentages were used to assess validity, the ability of the scheme to capture the free-text CCs. Kappa scores were used to compare the two independent analyses of the July 2006 data to measure agreement and reliability. Chisquared analysis was used to compare the data from July and February in order to measure the impact of seasonal variability on the validity of the scheme.

\section{Results}

July 2006 study period

Of 512 first CCs, 493 were interpretable and were used for study analysis. For the July 2006 study period, CCs were grouped into 8 major categories and 44 subcategories; 96.3\% CCs were categorized. The major categories and their proportions were: pain $32.3 \%$, injury $26.6 \%$, infection $15 \%$, psychiatric $8.1 \%$, miscellaneous $6.1 \%$, nurse only visit $5.5 \%$, eye $4.3 \%$ and rash $2.2 \%$.

The unweighted kappa score comparing the two independent analyses for the July study period was 0.89 (95\% confidence interval: 0.86-0.93).

February 2007 study period

Of 483 first CCs, 454 were interpretable and analysed during February; $96.9 \%$ were categorized into the 8 original major categories. The major categories and their proportions were: pain $30 \%$, injury $24 \%$, infection $21 \%$, psychiatric $10 \%$, miscellaneous $6 \%$, nurse only visit $3 \%$, eye $2.6 \%$ and rash $2 \%$. Refinements added 6 additional subcategories (blunt force injury to trunk, blunt force injury to head or neck, transplant, epistaxis, hardware problem and medication refill) (Table 1).

\section{July-February comparison}

There was no significant difference for the distribution of the eight major categories between July 2006 and February 2007 ( $p=0.13)$.

Some important clinical subcategory differences were found in the major category of infection: respiratory infections were more common in February than in July. Respiratory infections comprised $63.9 \%$ of all infections in February and $44.5 \%$ of all infections in July. Abscesses and other skin infections were more common in July, comprising $36.4 \%$ of all infections in July and $15.4 \%$ of all infections in February. Bites, burns, lacerations and punctures were more common in July ( $46 \%$ of all injuries) than in February (21\% of all injuries). Blunt force extremity 
Table 1 Chief complaint, major and subcategories

\begin{tabular}{|c|c|}
\hline Major category & Subcategory \\
\hline Pain & Ear pain \\
\hline Pain & Jaw pain \\
\hline Pain & Dental pain \\
\hline Pain & Headache or migraine \\
\hline Pain & Neck or shoulder or upper extremity pain \\
\hline Pain & Knee pain \\
\hline Pain & Hip or lower extremity pain \\
\hline Pain & Foot pain \\
\hline Pain & Chest or side pain \\
\hline Pain & Abdominal pain \\
\hline Pain & Back pain \\
\hline Pain & General muscle or joint pain \\
\hline Injury & Animal exposure concerning for rabies \\
\hline Injury & Insect bite or sting \\
\hline Injury & Blunt force injury to head or neck \\
\hline Injury & Blunt force injury to trunk \\
\hline Injury & Blunt force injury to arm or shoulder \\
\hline Injury & Blunt force injury to wrist or hand \\
\hline Injury & Blunt force injury to leg or hip \\
\hline Injury & Blunt force injury to ankle or foot \\
\hline Injury & Fall \\
\hline Injury & Low-speed collision \\
\hline Injury & Minor burn \\
\hline Injury & Puncture wound \\
\hline Injury & Laceration to face or scalp \\
\hline Injury & Laceration to upper extremity \\
\hline Injury & Laceration to lower extremity \\
\hline Injury & Wound or incision complication \\
\hline Injury & Wound or infection follow-up \\
\hline Infection & Abscess \\
\hline Infection & Dermatitis suspicious for infection \\
\hline Infection & Fever \\
\hline Infection & Pharyngitis symptoms \\
\hline Infection & Respiratory infection symptoms \\
\hline Infection & Urinary tract infection or gynaecological symptoms \\
\hline Psychiatric & Alcohol or drug problem without suicidal ideation \\
\hline Psychiatric & $\begin{array}{l}\text { Depression without suicidal ideation } \\
\text { or substance abuse }\end{array}$ \\
\hline Psychiatric & Suicidal ideation/attempt or homicidal ideation \\
\hline Psychiatric & Other mental health issue \\
\hline Miscellaneous & Allergic reaction \\
\hline Miscellaneous & Dizziness or vertigo \\
\hline Miscellaneous & Masses or lumps \\
\hline Miscellaneous & $\begin{array}{l}\text { Nausea/vomiting/diarrhoea/constipation/rectal } \\
\text { bleeding }\end{array}$ \\
\hline Miscellaneous & Swelling of an extremity \\
\hline Miscellaneous & Swelling of the face \\
\hline Miscellaneous & Syncope \\
\hline Miscellaneous & Epistaxis \\
\hline Miscellaneous & Transplant \\
\hline Miscellaneous & Medication refill \\
\hline Miscellaneous & Hardware problem \\
\hline \multicolumn{2}{|l|}{$\begin{array}{l}\text { Nurse only } \\
\text { visit }\end{array}$} \\
\hline \multicolumn{2}{|l|}{ Eye } \\
\hline Rash & \\
\hline
\end{tabular}

injuries were more common in February (26\% of all injuries) than in July (13\% of all injuries). Otherwise, the distributions of CCs between July and February were similar.

\section{Discussion}

This study demonstrates that an EDFT rotation for medical students can be organized using CCs that are encountered in actual practice. We are using this categorization as we develop a CC-based curriculum for our EDFT medical student rotation. Any educational curriculum is designed to teach the learner what he or she needs to know about the subject. It must be focused enough so that educators can cover the topics, yet broad enough to meet the EDFT educational goals. We feel that this eight major category system (pain, injury, infection, psychiatric, eye and rash) can serve as a starting point for an EDFT curriculum and allows easy breakdown into smaller subcategories. It is not all-inclusive, but can be expanded to incorporate EDFT CCs resulting from different triage practices and patient populations.

The following examples illustrate how a CC-based curriculum is different from a diagnosis-based curriculum. In diagnosis-based curricula, a student may be taught about the radiologic findings and treatment of a Lisfranc fracture of the foot. Using a CC-based curriculum in the category of 'injury (ankle/foot)', the student will be taught the process of evaluation of the complaint 'jumped off a ladder and hurt my foot' - a process that must consider factors such as exactly how the patient got injured, the implications of the mechanism of injury to the body, patient co-morbidities that can affect the injury or its prognosis, the associated injuries that should be anticipated and the proper diagnostics or examination points to confirm or eliminate elements of the differential diagnosis. In our study, $32 \%$ and $30 \%$ of visits were for the CC category of 'pain' in July and February, respectively. CC-based curricula should focus on the assessment of pain using a variety of pain scales, identifying individual risk factors for oligoanalgesia and, most importantly, translating these aspects into timely and effective ED and aftercare pain management [3].

Medical education usually focuses upon diagnosis-based pathophysiology. However, diagnoses do not reflect severity of the condition, mechanism of illness or injury, or comorbidities, and can vary with the diagnosis terminology preferences of the physician [4]. Educational curricula that typically discuss diagnoses rather than CCs are especially limited when applied to the ED where no information other than the $\mathrm{CC}$ is available at the start of the patient encounter, and the evaluation must be focused on the CC. In addition, curricula that list conditions to be mastered may not 
adequately reflect the types of complaints seen in day-today practice [5].

EDs are typically divided into care areas based upon acuity, for efficiency and staffing. Worldwide, the EDFT is a rich and efficient environment for learning, teaching and clinical practice [6,7], even in smaller sized EDs [8]. Students interested in specialties besides emergency medicine can benefit from exposure to the more common, less acute problems seen in the EDFT. For the first 2 years of our Department's EDFT offering to meet the medical school's ambulatory care rotation requirement (20062008), medical students interested in internal medicine, family practice, anaesthesia, psychiatry, radiology, urology, as well as emergency medicine, selected the EDFT rotation. For most students, this experience would be their only exposure to the world of emergency medicine and the more mundane human ailments. In addition, EDFT provides exposure to important elements such as history taking, physical examination, procedural skills and radiographic interpretation, which are sometimes deficient in interns [9].

Using the $\mathrm{CC}$ to develop a systematic approach to learning is effective when applied to common conditions that the student expects to see on a daily basis. However, analysing the $\mathrm{CC}$ is difficult due to the variety of presenting complaints, the lack of a standardized terminology or categorization system for $\mathrm{CC}$ and the common use of free-text entry to describe a patient's CC.

We arbitrarily selected 10 days of visits, in one summer and one winter month, to obtain about $500 \mathrm{CCs}$ for each study period. Census and admission rate do not vary from week to week in our EDFT. The number of CCs was limited to 500 because the lack of a controlled vocabulary or standardized system for CC required manual categorization. Several studies have used computer text-parsing algorithms to change free-text chief complaints into a coded scheme [10-13]. In these studies, most chief complaints were codable, but only post hoc. These studies concluded that coded CCs could improve syndromic surveillance, communication, ED technology support, inter-hospital evaluation and medical education. While improved medical education is one of the goals listed in these studies, education was not the primary focus for developing a CC coding system. Furthermore, the best scheme for a coherent educational curriculum may not be the same as the best scheme for outbreak detection or research.

\section{Limitations}

This system only applies to the EDFT and would not accurately reflect what learners would see in the entire ED. It should be used as a starting point, not as an all-inclusive list. However, the assessment and care of lower acuity problems is an important part of overall medical education, not just emergency medicine education, and this information could be used in developing part of the curriculum for an entire ED rotation. Our results are limited by location and geography as this was only performed at an academic ED in central North Carolina, and we are unsure about local, regional or global differences in $\mathrm{CCs}$ or patient populations. $\mathrm{CC}$ analysis in EDFT was limited by triage procedures which could be unique to our institution. For example, patients with mental health complaints who are felt by the triage nurse to not be dangerous to themselves or others are triaged to EDFT. Triage errors, or the use of EDFT in the case of ED overcrowding, could result in triage of more acute patients into EDFT. We attempted to address seasonal variability to ensure that students rotating at different times of the year would have similar clinical experiences based upon $\mathrm{CC}$, and the scheme performed well, but it has only been tested during the months of July and February.

\section{Conclusions}

This study describes a valid and reliable categorization scheme for free-text CCs in the EDFT of an academic ED. The scheme can guide curriculum development for learners in an academic setting, based upon actual anticipated clinical encounters that begin with the patient's reason for visit, or $\mathrm{CC}$, rather than upon a diagnosis established at the completion of the encounter.

Acknowledgements Matthew Frederick, D.O., assisted with the reliability study for July 2006 chief complaints while a senior medical student at the University of North Texas College of Osteopathic Medicine. Laura Zimmerman, Ph.D., M.D., assisted with the validity study for February 2007 chief complaints and assisted in refinement of categories while a senior medical student at the University of North Carolina. Mingrui Liu, Ph.D., performed statistical analysis.

Authors' contributions Adam R. Kuykendal, M.D. developed the categorization scheme and had primary responsibility for analysing data and preparing the manuscript.

Judith Tintinalli, M.D., M.S. had primary responsibility for the study concept, developing methodology and editing the manuscript.

Kevin Biese, M.S., M.D.: analysis and interpretation of data; review and editing of the manuscript.

Conflicts of interest None.

\section{References}

1. 2005) Emergency Severity Index v.4. In: AHRQ Publication No. 05-0046-2. http://www.ahrq.gov/research/esi. Accessed 11 Jan 2008

2. Hulley SB, Cummings SR, Browner WS, Grady D, Hearst N, Newman TB (2001) Designing clinical research, 2nd edn. 
Lippincott Williams \& Wilkins, Philadelphia, Chap. 4, Planning the measurements: precision and accuracy, $\mathrm{p} 44$

3. EMPainline. http://www.empainline.org/bi_painline/about.do. Accessed 26 Feb 2008

4. Feinstein AR (1988) ICD, POR, and DRG: unsolved scientific problems in the nosology of clinical medicine. Arch Intern Med 148:2269-2274

5. Manthey D, Coates WC, Ander DS et al (2006) Report of the Task Force on National Fourth Year Medical Student Emergency Medicine Curriculum Guide. Ann Emerg Med 47(3):e1-e7

6. Kwa P, Blake D (2008) Fast track: has it changed patient care in the emergency department. Emerg Med Australas 20:10-15 (Epub 12 Nov 2007). Accessed 11 Jan 2007

7. Rehmani R, Horain A (2007) Trends in emergency department utilization in a hospital in the Eastern region of Saudi Arabia. Saudi Med J 28(2):236-240

8. O'Brien D, Williams A, Blondell $\mathrm{K}$ et al (2006) Impact of streaming "fast track" emergency department patients. Aust Health Rev 30(4):525-532
9. Bansal PK, Saoji VA, Gruppen LD (2007) From a "generalist" medical graduate to a "specialty" resident: can an entry-level assessment facilitate the transition? Assessing the preparedness level of new surgical trainees. Ann Acad Med Singapore 36 (9):719-724

10. Aronsky D, Kendall D, Merkley K et al (2001) A comprehensive set of coded chief complaints for the emergency department. Acad Emerg Med 8(10):980-989

11. Day FC, Schriger DL, La M (2004) Automated linking of free-text complaints to reason-for-visit categories and International Classification of Diseases diagnoses in emergency department patient record databases. Ann Emerg Med 43:401-409

12. Thompson DA, Eitel D, Fernandes CMB, Pines JM, Amsterdam J, Davidson SJ (2006) Coded Chief Complaintsautomated analysis of free-text complaints. Acad Emerg Med 13:774-782

13. Travers DA, Haas SW (2004) Evaluation of Emergency Medical Text Processor, a system for cleaning chief complaint text data. Acad Emerg Med 11:1170-1176 